\title{
Uso de internet por los médicos colegiados de Valencia: un estudio de viabilidad de la Biblioteca Médica Virtual del Colegio Oficial de Médicos de Valencia
}

\section{Artículo}

\author{
Por Aurora González Teruel, María F. Abad García, Lluís Sanjuán Nebot, \\ Julia Campón Gonzalvo y Lourdes Castillo Blasco
}

\begin{abstract}
Resumen: Cuando se pretende establecer un nuevo servicio de acceso a la información distribuida en internet es necesario determinar cuál es el uso real para los propósitos que se pretenden ofrecer y cuáles son las dificultades a las que se enfrentan los usuarios potenciales a los que va dirigido este servicio. Se presentan los resultados de una investigación cuyo propósito ha sido conocer el nivel de uso de internet del colectivo de médicos colegiados de la provincia de Valencia para poner a su disposición un nuevo servicio, la Biblioteca Médica Virtual del Colegio Oficial de Médicos de Valencia.
\end{abstract}

Palabras clave: Uso de la información, Estudios de uso, Bibliotecas virtuales especializadas, Bibliotecas médicas virtuales.

Title: Internet use by affiliated doctors from Valencia: a study of viability of the Virtual Medical Library of the Official Medical Association of Valencia

Abstract: When trying to establish a new service for access to information distributed via the internet, it is necessary to determine the actual use of what is to be offered, as well as the difficulties encountered by potential users of this service. This article presents the results of a study to determine the level of internet usage among medical doctors affiliated with the provincial medical association in order to make available to them a new service: the Virtual Medical Library of the Official Medical College of Valencia.

Keywords: Information usage, Use studies, Specialized virtual libraries, Medical virtual libraries.

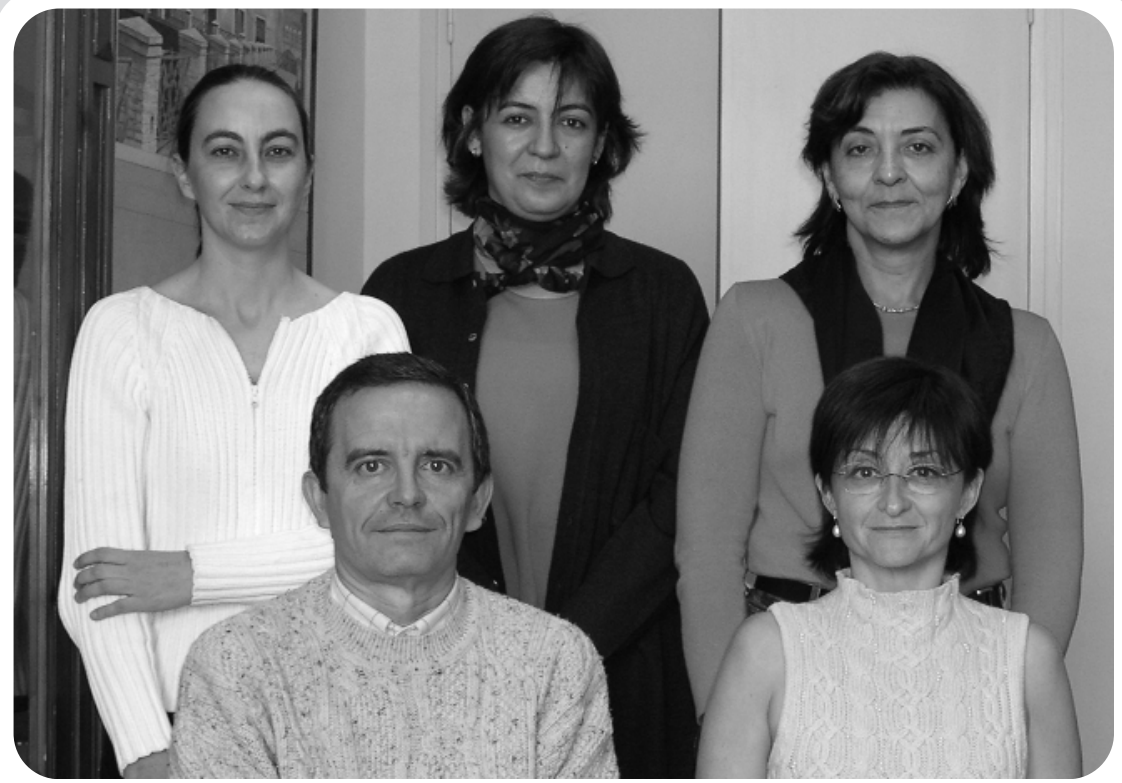

De izquierda a derecha y atrás a delante: Lourdes Castillo, documentalista de Rtwv, profesora asociada de biblioteconomía y documentación del Departamento de Historia de la Ciencia y Documentación de la Universitat de València; Aurora González, profesora titular de biblioteconomía y documentación del Departamento de Historia de la Ciencia y Documentación de la Universitat de València; Julia Campón, bibliotecaria de consorcio Hospital General Universitario de Valencia, profesora asociada de biblioteconomía y documentación del Departamento de Historia de la Ciencia y Documentación de la Universitat de València; Lluís Sanjuán, profesor titular de estadística y demografía, director de la Escuela Universitaria de Enfermería, Universitat de València; Francisca Abad, doctora en Medicina, profesora titular de biblioteconomía y documentación del Departamento de Historia de la Ciencia y Documentación de la Universitat de València.

González Teruel, Aurora; Abad Gar-

cía, María F.; Sanjuán Nebot, Lluís; Campón Gonzalvo, Julia; Castillo Blasco, Lourdes. "Uso de internet por los médicos colegiados de Valencia: un estudio de viabilidad de la Biblioteca Médica Virtual del Colegio Oficial de Médicos de Valencia". En: El profesional de la información, 2004, marzo-abril, v. 13, n. 2, pp. 100-106.

\section{Introducción}

Para los profesionales de la información es evidente que desde hace unos años internet constituye una importante fuente de información profesional y tam- bién una vía para la provisión de nuevos servicios de información. Según las últimas cifras del Estudio General de Medios ${ }^{1}$, el porcentaje de usuarios es del $27,4 \%$, dos puntos más que en el anterior período de febrero-marzo. Ello supone que en España hay 
9.652.000 internautas. El hogar sigue siendo el lugar desde donde se conecta principalmente $(62,4 \%)$, seguido del centro de trabajo y de la universidad. El servicio más utilizado continúa siendo la navegación y consulta de páginas web, seguido por el correo electrónico. El porcentaje de usuarios que hacen transferencia de ficheros $(29,9 \%)$ se mantiene estable, con ligeros descensos desde 2000.

\section{«En el entorno de los profesio- nales médicos las facilidades para el acceso a internet son cada día mayores y su uso tan- to en el medio social como pro- fesional está cada vez más arraigado»}

Por sexo, los hombres siguen siendo mayoritarios en la Red española ya que suponen un 58,5\% del total. En 1997 eran el 66,9\%. Por edades, el grupo mayoritario se sitúa entre los 25 y 34 años $(29,1 \%)$. Detrás se encuentran los de 35 a $44(18,8 \%)$ seguidos muy de cerca por los que tienen entre 14 y $19(18,6 \%)$ y los de 20 a $24(18,4 \%)$. Los mayores de 65 años siguen siendo un colectivo alejado de la Red. Por estrato social los ciudadanos de clase media son los más que más presencia tienen en internet $(43,5 \%)$ seguidos de los de media alta $(22,6 \%)$.

Una de las series de encuestas más extensa y fiable de la utilización de internet para localizar información médica la realiza periódicamente la Health on the Net Foundation $(H O N)$. Según la última realizada (junio de 2002) y contestada por un total de 2.621 profesionales, corresponden a Europa un 29\%, a América del Norte el $43,5 \%$ y el resto se reparte entre países de América del Sur, Asia, Oceanía, Oriente Medio y África. Según los resultados ofrecidos los usuarios buscan descripción de enfermedades, literatura médica, protocolos y grupos de trabajo, preferentemente.

Otra encuesta realizada por la consultora internacional The Boston Consulting Group ${ }^{2}$ (BCG) a 400 especialistas ofrece varias conclusiones importantes sobre el uso que hacen los médicos de la Red. El 89\% de los encuestados se conectaba como media ocho horas a la semana. Los autores del informe aseguran que la cifra es muy positiva sobre todo si se tienen en cuenta otros datos, como que el $90 \%$ de los especialistas la usa para recopilar información clínica y el $80 \%$ para leer artículos científicos. La formación médica continuada $(45 \%)$ o las conferencias online $(31 \%)$ cada vez tienen más aceptación. Entre los distintos servicios disponibles, los médicos encuestados consideran que la posibilidad de consultar historias clínicas mediante el uso del ordenador personal, prescribir fármacos, co- municarse con los pacientes a través del e-mail o controlar su estado a distancia hacen de internet una herramienta muy poderosa.

Respecto a la búsqueda de información por parte de los médicos, el sondeo del $B C G$ describe un patrón de comportamiento muy claro: al contrario que los pacientes, que acostumbran a no ser fieles a ningún portal específico y que acceden a la información que les interesa a través de buscadores generales, más de dos tercios de los especialistas que participaron en la encuesta visitan habitualmente entre dos y cinco en sitios concretos. Los preferidos son el líder de la información sanitaria dirigida a usuarios no especializados (WebMD), el principal portal de contenidos médicos de la Red (Medscape) y Physician's Online.

http://www.webmd.com

http://www.medscape.com

http://www.physiciansonline.com

La gran cantidad de páginas web con información biomédica, tanto nacionales como internacionales, así como el propio comportamiento de los profesionales de la medicina, han dejado patente la necesidad de que los profesionales de la información se ocupen de evaluarla y organizarla, con el objetivo de hacer asequible de forma racional la ingente cantidad de material diseminado por toda la web. En el entorno de los profesionales médicos las facilidades para el acceso a internet son cada día mayores y su uso tanto en el medio social como profesional está cada vez más arraigado.

Sin embargo, pese a esta percepción de uso, cuando se quiere establecer un nuevo servicio de forma exclusiva por esta vía es necesario determinar cuál es el uso real de internet para los propósitos que se pretende servir y cuáles son las dificultades a las que se enfrentan los usuarios potenciales a los que va dirigido este servicio cuando la utilizan, ya que esto condicionará en gran medida su viabilidad.

La necesidad de conocer exactamente el uso de este nuevo medio ha llevado a la realización de encuestas tanto de ámbito general (Ucla, 1999; Dryburgh, 2001) como enfocadas a colectivos determinados, entre ellos el de los médicos (Chi-Lum; Durkin, 1999; Casino, 1999; Kalsman; Acosta, 2000; Tatsumi et al., 2001; Harris Interactive, 2001, 2002). Pese a la utilidad que como marco de referencia puede tener conocer los resultados de estos estudios, no cabe duda que para el desarrollo de productos concretos es necesario disponer de datos acerca del entorno en el que este producto se quiere poner en marcha.

Conocer el nivel de uso de internet del colectivo de médicos colegiados de la provincia de Valencia ha sido el propósito de la investigación cuyos resultados se presentan aquí. El contexto en el que se realiza esta in- 


\section{Encuesta}

I.- Por favor, marque la casilla que refleje lo mejor posible su situación actual.

-No tengo acceso a un ordenador.

- Tengo acceso a ordenador pero

lo utilizo en escasas ocasiones.

- Uso ordenador y puedo acceder a la Red.

- Uso ordenador pero no puedo acceder a la Red.

-Otros (especificar).

2.- ¿Ha utilizado (usted o alguien por usted) alguna vez internet? -Sí, yo mismo.

- Sí, alguien en mi nombre.

-No (por favor indique los motivos y salte a la pregunta siguiente).

3. - Por favor, marque la frase que más se aproxime a lo que usted siente respecto a sus conocimientos de internet.

-No sé nada de internet y no tengo curiosidad por conocer más.

-No sé mucho acerca de internet pero me gustaría conocer más.

- Me siento seguro o bastante seguro utilizando internet.

-Otros (especificar).

4. - Desde dónde suele acceder habitualmente a internet.

- Casa.

-El trabajo.

- Universidad (si no es su trabajo).

-En cualquiera de los anteriores indistintamente.

-Otros (especificar).

5. - ¿Qué navegador es el que utiliza habitualmente para navegar por internet?

-Netscape.

-Explorer.

-Otro.

6. - ¿Dispone de cuenta de correo electrónico?

7. - ¿Con que frecuencia aproximada utiliza internet?

- Todos los días.

-Dos o tres veces por semana.

- Una vez por semana.

- Una vez cada quince días.

_Una vez al mes.
-Otros (especificar).

8. - Por término medio, cuando se conecta ¿cuánto tiempo invierte en el uso de internet?

- Menos de una hora.

-Entre una y tres horas.

-Entre tres y cinco horas.

-Otros (especificar).

9. - Por favor, señale con qué frecuencia tiene alguno de los siguientes problemas al utilizar internet para buscar información.

a) No soy capaz de encontrar la información que busco.

b) No soy capaz de guardar un documento que me interesa.

c) No soy capaz de encontrar una página que sé que existe.

d) No soy capaz de guardar una imagen que me interesa.

e) No puedo determinar dónde he estado y dónde quiero ir.

f) No soy capaz de visualizar archivos pdf.

g) No soy capaz de descargar y recuperar un archivo comprimido.

h) Otros.

10. - Habitualmente ¿cómo suele localizar nuevas páginas web?

- Libros y revistas.

-Buscadores.

—Amigos.

- Portales sanitarios.

—Enlaces de otras páginas.

-Otros (especificar).

II. - Por favor, señale la frecuencia con la que usted suele utilizar internet con los siguientes propósitos:

a) Correo electrónico.

b) Educación en general.

c) Lectura de la prensa electrónica.

d) Buscar información relacionada con mi actividad médica.

e) Compras.

f) Transacciones electrónicas.

h) Juegos y actividades de ocio (chat...).

i) Reserva de billetes, consulta de callejeros...

j) Otros.

12. - Por favor, señale de entre las siguientes categorías aquella que mejor refleja el área de su actividad profesional para la que utiliza internet con mayor frecuencia.

-Buscar datos concretos (fármacos, constantes, etc.).

- Buscar información para la toma de decisiones clínicas.

- Comunicación con colegas.

- Buscar información para la elaboración de informes, ponencias, artículos...

-Buscar información para la actualización de conocimientos.

—Otros (especificar).

13. - ¿De qué modo valora usted la iniciativa del Colegio de crear una biblioteca virtual?

-Muy positiva.

-Positiva.

-Indiferente.

-Negativa.

-Muy negativa.

14. - ¿A que tipo de recursos considera usted que la biblioteca debería dar acceso?

-Diccionarios, atlas, clasificaciones...

- Información estadística (demografía, mortalidad).

- Bases de datos bibliográficas de acceso gratuito.

- Bases de datos bibliográficas de pago.

- Revistas y publicaciones electrónicas (libros, dossiers, informes...).

-Catálogos y directorios.

-Páginas institucionales.

-Noticias médicas.

-Enlaces de interés por especialidad.

-Buscadores y portales sanitarios. -Otros (especificar).

15. - ¿Qué requisitos cree usted que debería reunir de forma imprescindible la futura Biblioteca Médica Virtual del Colegio de Médicos de Valencia?

a) Organización por especialidades.

b) Organización por tipo de recursos.

c) Posibilidad de búsqueda avanzada.

d) Descripción de los recursos.

e) Documentos de ayuda para el 
uso de determinados recursos.

f) Servicios de consultoría.

g) Servicios de reprografía de artículos de interés.

h) Inclusión del mayor número posible de enlaces de interés.

i) Inclusión de los enlaces de calidad.

j) Otros.

16. - ¿Cuál es su especialidad?

17. - ¿Dónde desempeña su trabajo?

-Hospital.

- Centro de especialidades.

- Centro de salud.

- Consultorio zona rural.

-Empresa.

- Consulta privada.

-En paro.

-Jubilado.

-Otros.

18. - Su residencia habitual se encuentra ubicada en:

-Municipio $<5.000$ habitantes.

-Municipio 5.001 - 10.000

habitantes.

-Municipio 10.00I - 25.000

habitantes.

-Municipio > 25.00l habi-

tantes.

19. - Sexo

- Hombre.

-Mujer.

20. - Por favor, indique el grupo de edad al que pertenece

—de 25 a 30 años.

-de 31 a 35 .

-de 36 a 40.

-de 41 a 45.

- de 46 a 50.

- de 51 a 55 .

一de 56 a 60.

一de 61 a 65.

-Mayor de 66 años.

21. - Por favor indique las páginas o recursos que usted considera que son más relevantes para su especialidad. vestigación es el desarrollo de la $B i$ blioteca Médica Virtual del Colegio de Médicos de Valencia (Comv).

La puesta en marcha de esta biblioteca responde a un doble objetivo: Por una parte, paliar la carencia de infraestructuras de información del Colegio de Médicos de Valencia que le permita cumplir con una de sus tareas fundamentales: servir de apoyo y motor para la formación continuada y para la investigación de sus colegiados. Además, aprovechar las posibilidades que brindan las nuevas tecnologías para prestar este servicio de un modo uniforme y equitativo a todos sus colegiados, sin distinción de lugar de residencia, ubicación y categoría laboral o cualquier otra condición.

La Biblioteca Médica Virtual es un proyecto desarrollado por el Instituto de Historia de la Ciencia y Documentación López Piñero (IHCyD, Csic-Universitat de València) para poner a disposición de los médicos de la provincia información relevante para la toma de decisiones en la práctica

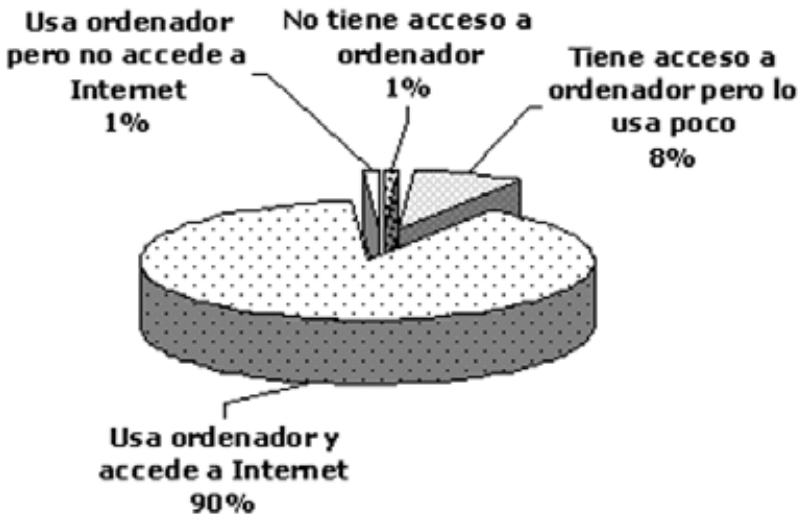

Figura 1. Accesibilidad a internet y uso del ordenador

clínica, la investigación, la formación continuada, la docencia y la gestión.

La biblioteca permite el acceso remoto a través de internet a un conjunto de recursos y servicios, tradicionalmente prestados por bibliotecas, hemerotecas y centros de documentación. Desde su puesta en marcha en abril de 2002 (dos meses después de la realización de la encuesta) da acceso a dos tipos de recursos:

-Para la búsqueda y recuperación de información, entre los que se encuentran algunos de elaboración propia como la base de datos de recursos web médicos que actualmente contiene más de 4.000 fuentes de información médicas y productos comerciales, que es el caso del acceso online a bases de datos mediante la interfaz Ovid (Medline, PreMedline, Embase, Evidence Based Medicine Review y las revistas ACP journal club y Clinical evidence).

http://www.comv.es

- Recursos creados con la finalidad de agilizar las tareas de los médicos y de prestarles mayor grado de autonomía. En esta categoría se encuadran el servicio de asesoramiento para la obtención del documento primario y el apartado Mi escritorio, así como otros recursos de utilidad tales como el cajón de herramientas y el tablón de anuncios. Esta biblioteca cuenta con espacio específico para la comunicación con el usuario, desde el cual poder formular preguntas, sugerencias, mandar recursos o dar su opinión acerca de la satisfacción de este servicio mediante la cumplimentación de una encuesta.

\section{Material y método}

\section{Población.}

En enero de 2002 el número de médicos colegiados en el Comv ascendía a 10.450. Para la realización de este estudio se seleccionó una muestra aleatoria de 500 profesionales estratificada según edad, sexo y tenencia de cuenta de correo electrónico del Colegio. 


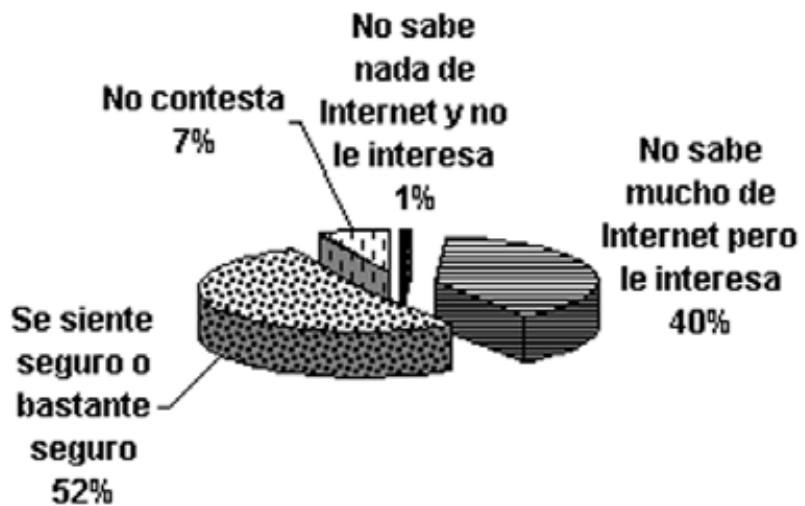

Figura 2. Conocimiento de internet

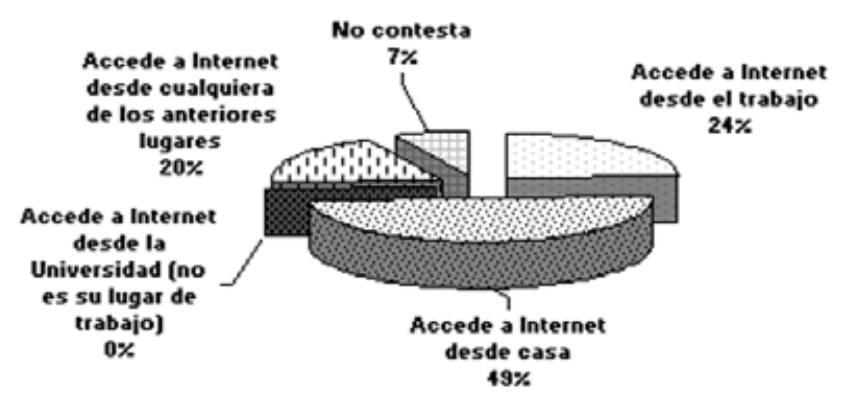

Figura 3. Lugar de conexión

Previo al diseño definitivo de la encuesta se realizó un estudio piloto para el que se utilizó una versión previa de la misma con el objetivo de que fuera cumplimentada por los presidentes de las sociedades científicas que acudieron al acto de presentación del proyecto el 3 de enero de 2002. Se cumplimentó un total de 20, acompañadas de sugerencias que mejoraban la redacción de las preguntas, la categorización o la inclusión de nuevas cuestiones.

La que se ha utilizado finalmente consta de 20 preguntas que versaban sobre: tenencia de ordenador, uso de la Red, experiencia en su uso, acceso, navegador utilizado, tenencia de cuenta de correo, frecuencia de uso, tiempo medio de conexión, problemas de utilización, modo de localización de páginas web, propósito de uso (general), propósito de uso con fines profesio-

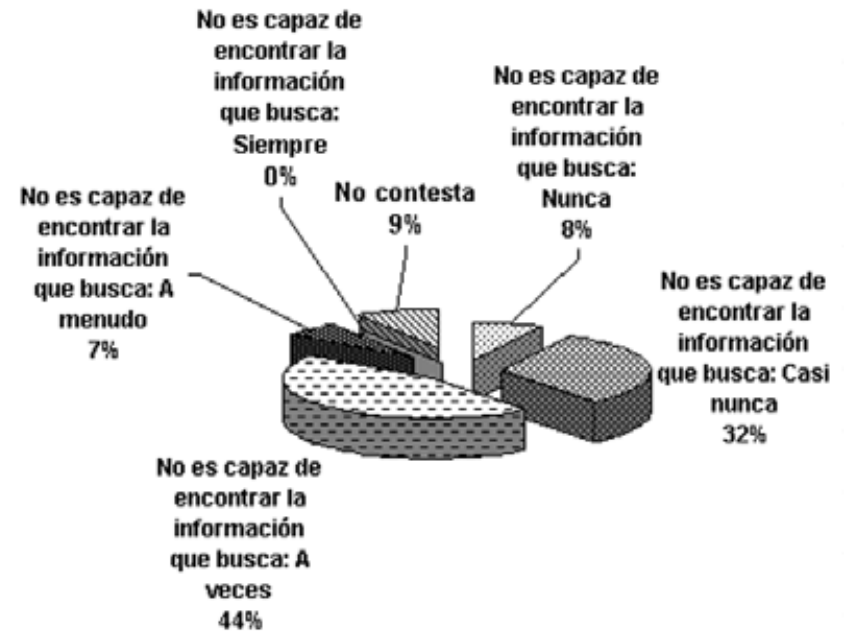

Figura 4. Problemas para encontrar la información buscada

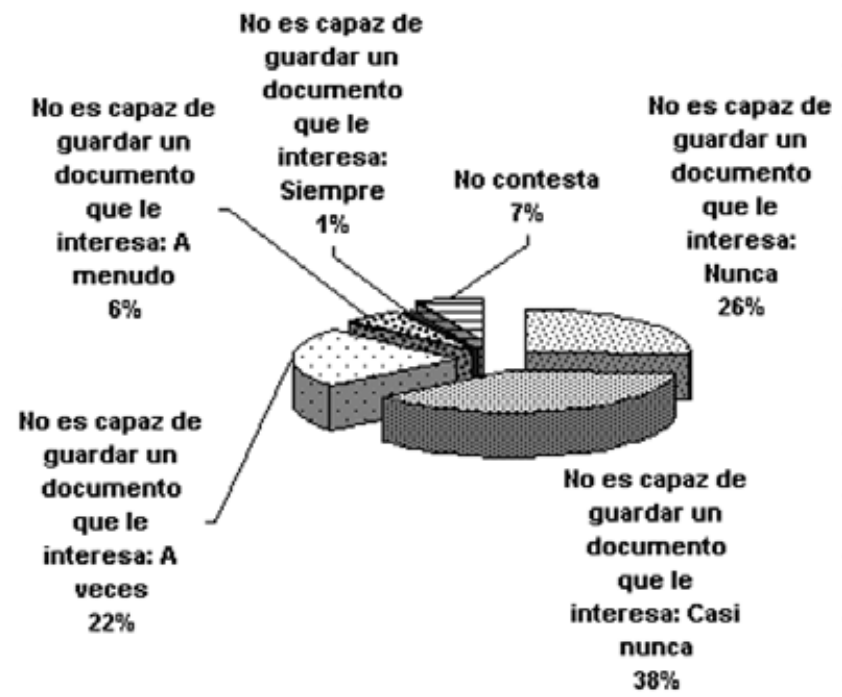

Figura 5. Problemas para guardar documentos

nales, valoración de la iniciativa del Comv de crear una biblioteca virtual, recursos a los que la biblioteca debería dar acceso y, por último, requisitos imprescindibles que debería cumplir. Adicionalmente se preguntaba acerca de las características personales (grupo de edad y sexo) y laborales (especialidad, lugar donde desempeña su actividad laboral).
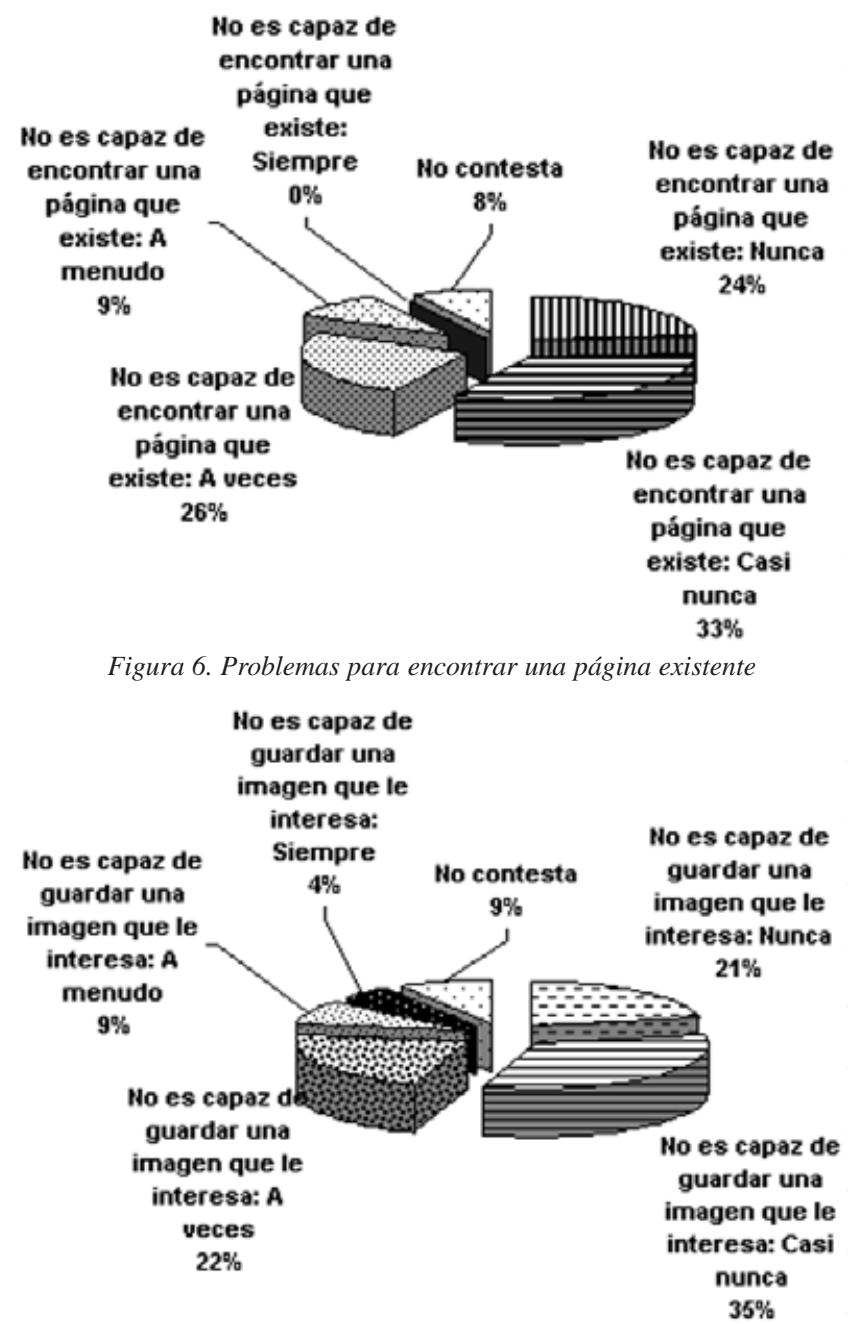

Figura 7. Problemas para guardar imágenes 

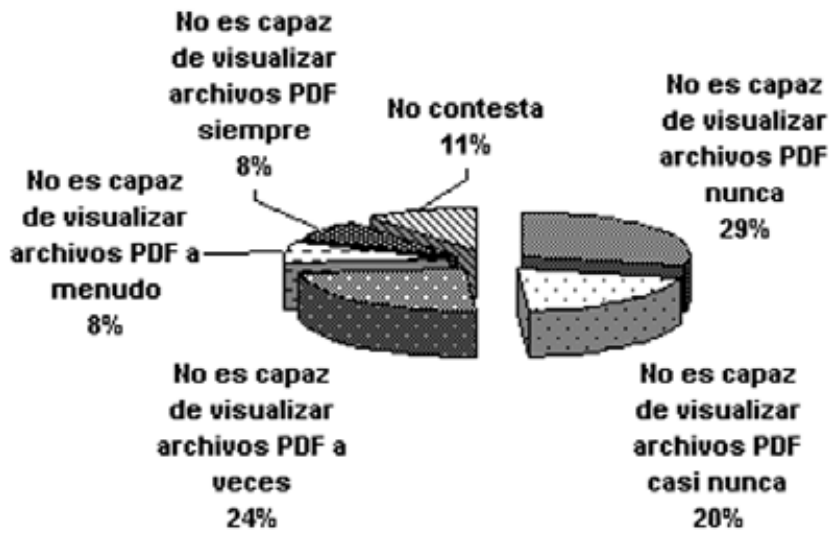

Figura 8. Problemas para usar archivos pdf

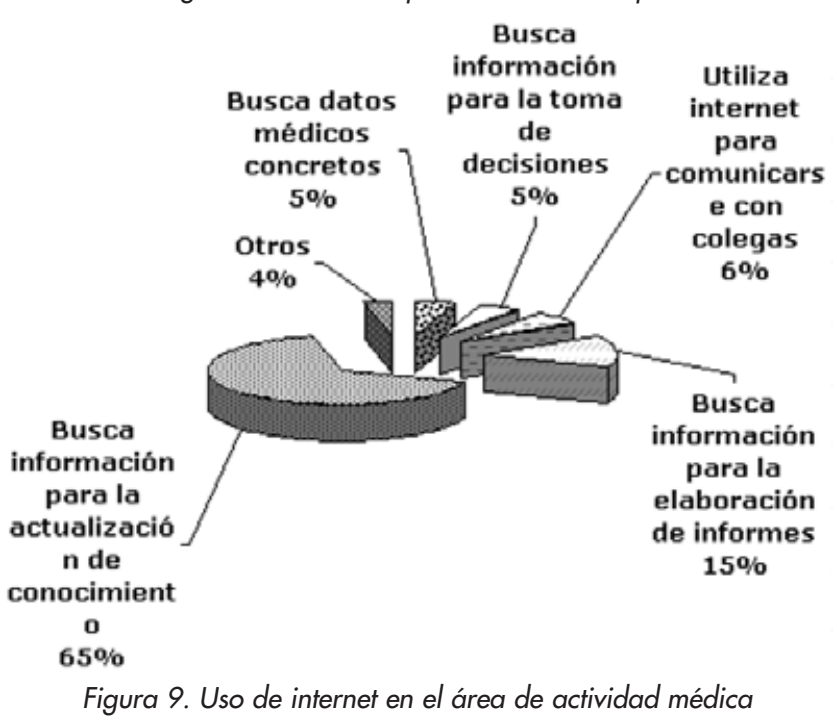

La encuesta se envió por correo acompañada de una carta explicativa, en la que además se solicitaba al médico que contestara en un plazo determinado de tiempo (21 días entre su envío y el cierre de recepción) y de un sobre con franqueo prepagado y dirección preimpresa para facilitar la respuesta.

\section{Resultados.}

Se recibieron 96 encuestas en el intervalo de tiempo especificado, lo que supone una tasa de respuesta del $19.2 \%{ }^{3}$. De éstas, el $68 \%$ pertenece al sexo masculino, el $26 \%$ al femenino y un $6 \%$ no contesta. En cuanto a los tramos de edad de los colegiados, el mayor porcentaje se encuentra en el de los 41 a 45 años (37\%), seguido del de 56 a 60 años 8 (25\%). El 77\% de los colegiados reside en localidades de más de 25.000 habitantes. Los resultados de cada una de las preguntas realizadas son las siguientes.

Respecto al uso de ordenador y de internet (figura 1) el $90 \%$ de los médicos afirma que lo usa y que puede acceder a la Red; de éstos, un 73\% emplea como navegador el Internet Explorer.

Con relación a sus conocimientos de internet el $52 \%$ se siente seguro o bastante seguro (figura 2). El lugar más frecuente de conexión (figura 3) es el domicilio particular (49\%), seguido del puesto de trabajo (24\%). La periodicidad en el uso de la Red es diaria en el $44,4 \%$ de los casos y de al menos dos o tres veces por semana para un $30,3 \%$. Respecto al tiempo de conexión, un $61,1 \%$ invierte menos de una hora y el $36,7 \%$ entre una y tres horas.

La localización de información buscada, la metodología a seguir para guardar información encontrada o una imagen, la orientación durante la navegación, la visualización de archivos pdf o la posibilidad de descargar y recuperar un archivo comprimido plantea problemas frecuentemente entre un 7 y un $13,5 \%$ de los médicos encuestados (figuras 4 a 8 ).

«El mantenimiento y actualización, en especial de las bases de datos gratuitas y de las publicaciones electrónicas deben ser un objetivo prioritario en el futuro»

La frecuencia de utilización del correo electrónico es elevada. El 70\% de los médicos encuestados afirma utilizarlo a menudo. El uso más frecuente de internet está relacionado con la actividad profesional $(55,6 \%$ lo utiliza a menudo y un $18,9 \%$ declara hacerlo siempre) con la finalidad principal de buscar información para la actualización de conocimientos (63\%). La figura 9 muestra otros usos de internet en el área de actividad médica.

El $94,8 \%$ valora positiva, o muy positivamente, la iniciativa del colegio de la creación de la biblioteca

\section{El profesional de la información} está abierto a todos los bibliotecarios, documentalistas y otros profesionales de la información, así como a las empresas y organizaciones del sector para que puedan exponer sus noticias, productos, servicios, experiencias y opiniones.

Dirigir todas las colaboraciones para publicar a:

El profesional de la información Apartado 32.280 08080 Barcelona

Fax: +34-932 08I 97। epi@sarenet.es 
virtual. Los encuestados opinan que debe dar acceso sobre todo a: bases de datos de acceso gratuito (90\%), revistas y publicaciones electrónicas $(76,6 \%)$, enlaces de interés por especialidad $(76,6 \%)$ así como a portales y buscadores sanitarios $(66,6 \%)$.

\section{Conclusiones}

Las respuestas son indicativas de un uso suficiente de la Red y de la necesidad de un servicio como la biblioteca virtual que puede facilitar la localización de la información profesional distribuida en internet, de un modo racional, mediante la clasificación temática y la distinción por tipo de recurso. El mantenimiento y actualización, en especial de las bases de datos gratuitas y de las publicaciones electrónicas deben ser un objetivo prioritario en el futuro dadas las preferencias que se reflejan en este trabajo.

Nos movemos en una rama del conocimiento en la que la necesidad de actualización de la información está modificando las líneas de actuación de los centros de documentación médicos (hemerotecas, bibliotecas especializadas). También las editoriales han detectado, al igual que los encuestados, la necesidad de esa actualización constante a la que hacemos referencia.

La biblioteca virtual que estudiamos es un intento, limitado por condicionamientos económicos, de dar solución al problema planteado entre aquellos profesionales que no tienen posibilidades de acceder a un gran centro sanitario que cuenta con recursos necesarios en el área de la documentación. Por ello intentamos atender estas necesidades y especialmente el desarrollo de una hemeroteca online y de los recursos de información médica a los que en teoría se podría acceder desde un centro.

Otro de los objetivos debe ser facilitar a los colegiados conocimientos de microinformática a través de manuales electrónicos, tutoriales o de recursos que les permitan aprender a conocer los distintos tipos de ficheros electrónicos existentes, los programas para su lectura así como su manipulación para un aprovechamiento personal eficaz.

\section{Notas}

1. Publicadas en El país, jueves 17 de julio de 2003.

2. Disponible en inglés. Los principales datos se publicaron en El mundo salud del 19 de enero de 2002.

http://www.bcg.com

3. Las tasas de respuesta de trabajos similares varían entre un $87 \%$ en el estudio de Chi-Lum y un 21,6\% en el de Lacher

\section{Bibliografía}

Casino, G. "Médicos en internet". En: Escepticemia, 1999, junio, n. 18. Consultado en: 09-07-02.

http://db2.doyma.es/cgi-

bin/wdbcgi.exe/doyma/pescepticemia.plantilla?pident $=135$
Chi-Lum, B.; Durkin, R. M. "Physicians accessing the internet: the PAI Project". En: Jama, 1999, v. 282, n. 7, pp. 633-634.

Dryburgh, H. Changing our ways: why and how Canadians use the internet.

Consultado en: 25-07-02.

http://www.statcan.ca/english/research/56F0006XIE/56F0006XIE.pdf

Fernández, G.; Susi, Ch.; Alonso, M. Dos encuestas sobre conocimiento y uso de internet en médicos. Consultado en: 25-04-02. http://www.hup.es/ecl/nueva/otr/encu98/encu98.htm

Guardiola, Elena. "Los médicos europeos e internet". En: El profesional de la información, 2003, v. 12, n. 6, nov.-dic., pp. 494-497.

Gutiérrez Morán, S. H. "Necesidades informativas de los médicos que laboran en el municipio de Maracaibo". En: Mística (Metodología e impacto social de las tecnologías de la información y de la comunicación en América), 1999. Consultado en: 25-04-02.

http://www.funredes.org/mistica/castellano/ciberoteca/participantes/docup arti/esp_doc_30.html

Harris Interactive. "The increasing impact of eHealth on physician behavior". En: Health care news, 2001, noviembre, v. 1, n. 31. Consultado en: 09-07-02.

http://www.harrisinteractive.com/news/newsletters/healthnews/HI_Health CareNews2001Vol1_iss31.pdf

Harris Interactive. "European physicians especially in Sweden, Netherlands and Denmark, lead US in use of electronic medical records". En: Health care news, 2002, agosto, v. 2, n. 16. Consultado en: 10-10-02. http://www.harrisinteractive.com/news/newsletters/healthnews/HI_Health CareNews2002Vol2_Iss16.pdf

Health on the Net Foundation. "Excerpt of the $8^{\text {th }}$ HON's survey of health and medical internet users" Consultado en: 12-06-02. http://www.hon.ch/Survey/8th_HON_results.html

Kalsman, M. W.; Acosta, D. A. "Use of the internet as a medical resource by rural physicians". En: Journal of American board family practice, 2000 , v. 13 , n. 5 , pp. 349-352.

Lacher, D.; Nelson, E.; Bylsma, W.; Spena, R. Computer use and needs of internists: a survey of members of the American College of PhysiciansAmerican Society of Internal Medicine. Consultado en: 25-07-02. http://www.amia.org/pubs/symposia/D200043.pdf

Rozic-Hristovski, A. "Users information-seeking behavior on a medical library website". En: Journal of Medical Library Association, 2002, v. 90, n. 2 .

Tatsumi, H.; Mitani, H.; Haruki, Y; Ogushi, Y. "Internet medical usage in Japan: current situation and issues". En: Journal of medical internet research, 2001, v. 3, n. 1.

Ucla Center for Communication Policy. Internet Proyect. Consultado en: 25-04-02.

http://ccp.ucla.edu/pages/InternetStudy.asp

Aurora González Teruel, Departamento de Historia de la Ciencia y Documentación de la Universitat de València. Aurora.Gonzalez@uv.es

Maria F. Abad García, Departamento de Historia de la Ciencia y Documentación de la Universitat de València. Maria.F.Abad@uv.es

Lluís Sanjuán Nebot, director de la Escuela Universitaria de Enfermería, Universitat de València. lluis.sanjuan@uv.es

Julia Campón Gonzalvo, Departamento de Historia de la Ciencia y Documentación de la Universitat de València. campon_jul@gva.es

Lourdes Castillo Blasco, Departamento de Historia de la Ciencia y Documentación de la Universitat de València. M.Lourdes.Castillo@uv.es 